\begin{tabular}{ccc}
\hline International Journal of Engineering \&Technology, $7(4.24)(2018) 137-141$ \\
SPC & Website: www.sciencepubco.com/index.php/IJET \\
Research paper & Technology \\
\hline
\end{tabular}

\title{
Optimal Placement of DG for Optimal Reactive Power Dispatch Using PSO Algorithm
}

\author{
Dsnmrao $^{* 1}$, Dr.Niranjan Kumar ${ }^{2}$ \\ ${ }^{1}$ Assistant Professor, Department of Electrical \& Electronics Engineering, VFSTR University, Vadlamudi, A.P, India-522213 \\ ${ }^{2}$ Professor, Department of Electrical \& Electronics Engineering, National Institute of Technology Jamshedpur, India-831014 \\ *Corresponding author E-mail:dsnm.rao@gmail.com ${ }^{1}$,nkumar.ee@nitjsr.ac.in ${ }^{2}$
}

\begin{abstract}
In the safety and economic point of view, Reactive Power is the most problematic thing during the operation of the electrical network. Reactive Power supply completion has nonlinear, equality and inequality constraints. Proposed work is carried out, to find the solution for reactive power supply issue, Particle Swarm Optimization (PSO) process as well as MATPOWER 5.1 implement package are developed in this process. PSO is an excellent optimization technique that is also having effective finding ability. One of the best assets of PSO is that its capacity is fewer sensitive than complication of the independent function. MAT POWER 5.1 is an undeveloped basis MATLAB implement package, concentrating the power flow issues findings. Suggested technique diminishes active power damage in the conventional power system and decides the optimum location of a newly setup Distributed Generator (DG). The IEEE 14bus arrangement is utilized to find the performance and test outcomes shown the perfectness of the recommended method.
\end{abstract}

Keywords: Reactive Power; Particle Swarm Optimization (PSO), MATPOWER, Distributed Generator (DG), Real Power Loss

\section{Introduction}

Earlier, Optimum Power Flow (OPF) issue and real power is solved by loss formulae and various strategies. Reactive power is also being optimized by approximate methods. Later, many research works depict solution for OPF, Real and Reactive power flow precisely. In 1962, the objective function for OPF problem subjects to the equality and inequality constraints is formulated. Later extensions have been made evolved those provide very fast and accurate solutions even for practical large systems [1].

Electric power utility variations in power systems are usual hour to hour. Changes in power result in voltage variations. For reliable customer services, maintaining voltage levels in allowable range is one of the challenging tasks [2]. For safety and viable operation of power system, consideration of reactive power is mandatory. Now the issue is to assign reactive power to reduce the real power wastage and maintaining same voltages by sustaining the quantity of fairness and disparity constraints. Real power is related to equality constraints and on other hand unequal constraints are related to upper and lower voltages limits, capacity limit of different var sources like generators, shunt capacitor banks and transformer tap settings [3], [4].

In an electrical distribution system reactive power control is a critical task. Accurate reactive power control reduces the true power losses and maintains the system potential within the limits. Reactive power control can be done automatically or manual control by changing the tapings of the power transformer and shunt compensation. In the view of environmental considerations and shortages' of conventional fuels, inverter based distributed- generation $(\mathrm{dg})$ resources are playing a key role. To full fill electrical energy demand, Wind-turbine generator is one of the DG resource and it is meeting considerable power demand in the distribution system [5-6].

The optimum reactive power dispatch (ORPD) is the major issue and mainly effects the commercial and safety operation of power system. To solve OPF problem, we have different conventional methods like Direct Programming, Quadratic Programming and Newton Rapson based approaches and in this all conventional methods, distinct values are preserved as constant variables and smoothed off to nearest rate after optimization, thus mathematical calculations take place, also increases the objective function assessment and finally that all effects on convergence difficulty and limits the possibility of useful application [8]. Main theme of placing DG unit is to diminish losses and DG is located in primary distribution system. During the location and sizing problem of DG cannot consider the cost of it and other advantages. The capacity and location of DG established on single direct demand at topmost, where the losses are maximized [9].

Mainly detached task of ORPD tricky refers to diminish the conductive real power losses, by sustaining numerous fairness and disparity constraints. Suggested scheme obtains the issue of introducing the optimum DG category to be located by standings of locality and size, substance to inverse power flow controls, is observed through a Particle Swarm Optimization process (PSO) beneath innovative besides extra decontrolled perception. PSO is best Evolutionary Computation (EC) techniques, improved methodology, applied to several problems and the inventive technique is capable to maintain the constant state variables 
simply [5-7]. Furthermore, the technique can be extended to maintain constant as well as distinct variables simply.

\section{Optimal Reactive Power Dispatch}

All paragraphs must be justified alignment. With justified alignment, both sides of the paragraph are straight.

\subsection{Necessity of Optimal Reactive Power Dispatch}

In general ORPD is employed to enhance economy as well as safety power system operation, thus obtains a lot of consideration at present, the reason for the ORPD in a power system operation is to identify the finest standards of the regulating variables such as alternator voltage magnitudes, compensation devices and transformer tap setting positions to be switched. Main theme of OPRD problem is to reduce actual power losses voltage deviations and enhanced the voltage constancy of the arrangement [8].

\subsection{Need for Real Power Loss Minimization}

The most essential operating condition of consistent power systems is to keep the voltage within the acceptable limits to establish a good customer service feature. Sensitive power and voltage regulating issues have gained importance to establish a reliable quality of power supply with the least possible losses in the power system network [9-10].

An extended load for electric power, the deficient power generation and transmission efficiency forces the power system is being operated under focused on conditions. In the event that the power system network is worked in focused conditions then security of a power system network is under risk and may bring about voltage instability. The voltage insecurity has turned into a new challenge to the power system network operation besides planning. Lacking volatile power convenience or non-optimized reactive power flow be allowed to the power system network of instability action during heavy loaded conditions [11].

\section{Reactive Power Dispatch Problem Formulation}

In power system network, loads are changing continuously. To operate the power system network at the ideal and convenient state, the optimization of reactive power dispatch is to be conducted constantly. Thus appears to be good for network, but constant switching operations are not possible in practical applications. These operations won't carry additional capacity to the operator of the system then additionally hasten the era of the apparatus in power system network. Occasionally constant exchanging operations may even impend the protective operation of the network. Hence, the number of switching operations as well as tap positions changing operations is severely limited [12].

\subsection{Objective Function}

In power systems network, reactive power dispatch has manyt objectives. Thus can limit real power losses and obtains best voltage profile by using smallest capacitors also attains maximum economic return. This paper aims the reactive power disatch to get the minimum tangible power loss [11].

The Proposed system indicates that active power loss is equal to addition of the real power loss on each branch and its representation is as follows [13].

$P_{\text {loss }}=\sum_{K=1}^{N} g_{i j}\left(v_{i}^{2}+v_{j}^{2}-2 v_{i} v_{j} \cos \theta_{i j}\right)$
Where $\mathrm{N}$ : is branch numbers,

$\mathrm{g}_{\mathrm{ij}}$ : branchconductance between $\mathrm{i}$ and $\mathrm{j}$ buses,

$\mathrm{v} i$ : bus voltage at $\mathrm{i}$,

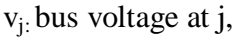

$\theta_{i j}$ : angle between $i$ and $\mathrm{j}$ buses

\subsection{Equality Constraints}

The equality constraints are nothing but power equivalence conditions which are specified by the following equations [12]

Pgi - Pdi - Vi $\sum V j(G i j \cos \theta i j+B i j \sin \theta i j)=0$

Qgi - Qdi $-\mathrm{Vi} \sum \mathrm{Vj}(\mathrm{Gij} \sin \theta \mathrm{ij}-\mathrm{Bij} \cos \theta \mathrm{ij})=0$

Where Pgi: generation of active power at bus $\mathrm{i}$

Pdi: plea of active power at bus $i$

Qgi: generation of reactive power at bus i

Qdi: plea of reactive power at bus i

Gij: conductance of communication line from $i$ bus to $j$ bus

Bij: susceptance of communication line from $i$ bus to $j$ bus

\subsection{Inequality Constraints}

The inequality functions ranges are nothing but voltage magnitudes, injecting of reactive power and transformer tap setting positions [14], are continuous and injecting of reactive power is discreate [15-16]. To manage the discrete values, the commonly used method views the constant standards at the initial optimization.Tthen after mapping the constant standards back to the distinct standards at the termination. In proposed article, distinct variables are perceived as constant variables initially besides keeping 3 decimal places at the search end [13].

$$
\begin{aligned}
& V_{i}^{\min }<V_{i}<V_{i}^{\max } \\
& t_{j}^{\min }<t_{j}<t_{j}^{\max }
\end{aligned}
$$

$Q_{g i}^{\min }<Q_{g i}<Q_{g i}^{\max }$

\section{Procedures for Particle Swarm Optimization (PSO) Based ORPD}

PSO having a multiple finding point based process which searches finest result through improving an objective task [4]. Every examining point is a mediator by a relative point. Every agent's location is characterized by $\mathrm{n}$ dimensional space and every measurement is combined with a velocity. This velocity indicates the agent's displacement rate. Every mediator tends to adjust its position from the current location $\boldsymbol{s}_{\boldsymbol{i}}^{\boldsymbol{k}}$, and from the current quickness for the following reiteration $\boldsymbol{v}_{\boldsymbol{i}}^{\boldsymbol{k}+\boldsymbol{1}}$ as shown in (7) and (8).

$$
\begin{aligned}
& s_{i}^{k+1}=s_{i}^{k}+v_{i}^{k+1} \\
& v_{i}^{k+1}=w_{k} v_{i}^{k}+c_{1} r_{1} \times\left(p_{\text {best }-i}-s_{i}^{k}\right)+c_{2} r_{2} \times\left(g_{\text {best }}-\right. \\
& \left.s_{i}^{k}\right)
\end{aligned}
$$

Where,

$v_{i}^{k}: \quad$ agent velocity $\mathrm{i}$ at iteration $\mathrm{k}$

$w$ : Inaction of mass

$c_{1}, c_{2}$ : Positive weight constants

$r_{1}, r_{2}$ : sum between zero and one randomly

$s_{i}^{k}: \quad$ Agent $\mathrm{i}$ at reiteration $\mathrm{k}$ of current point

$p_{\text {best }-i}$ : Specific finest of mediator $\mathrm{i}$ 


\section{$g_{\text {best }}:$ Finest of the set}

Principle optimization steps for the Particle Swarm Optimization (PSO) built reactive power communication as follows [17].

1. Load event data: In MATPOWER IEEE 14 buses arrangement information is kept in case14.m organizer. Clients can likewise make the customized instance via taking after sanctioned arrangement types of buses, branches and alternators.

2. Initialization: Arrange the absolute iteration value, particle total number, initial acceleration at random allow the fix of each particle in the design area. At that point assess the wellness of every unit and spare the worldwide finest well -known point and nearby finest well identified point of every unit.

3. Redesign the locations and speediness: Upgrading the location and speed of every unit. At that point check-up if the explanation violate the breaking point on the other hand not. On the off chance that the solution violates the breaking points, utilize the Exterior Penalty Function (EPF) strategy to penalize the desecrations.

4. Assess every unit: Add every particle location into the objective task to add the assessment rate.

5. Upgrade nearby finest well identified point: In the event that the present wellness value is littler than authentic super wellness value, upgrade the confined best well known point.

6. Upgrade worldwide best-known location.

Choose closing condition: Regulate, uncertainty the repetition had achieved the greatest repetition quantity. Uncertainty, close the optimization procedure besides design outcome; or else, iter=iter+1 then return to stage 3 .

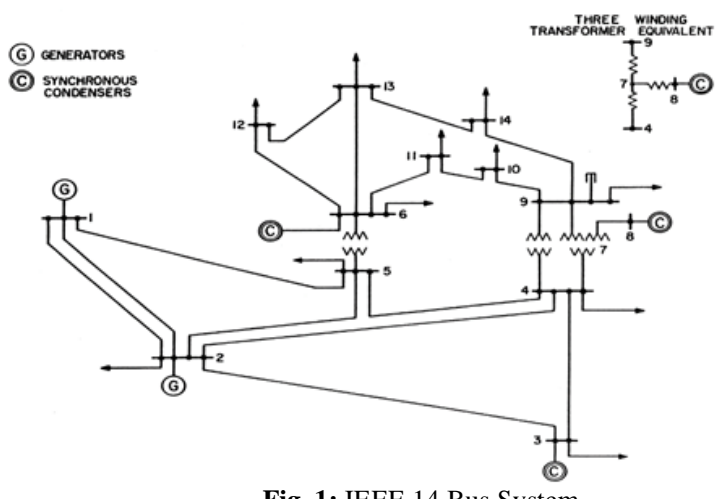

Fig. 1: IEEE 14 Bus System

\section{Simulation Results and Discussions}

Execution of the proposed strategy confirmed to IEEE 14 buses arrangement. Structure of 14 buses network is presented in Fig.1 [2].

Mainly two alternators are used in IEEE 14 buses arrangement. First alternator is connected at the slack bus and second alternator is connected at bus two and there are three synchronous condensers are situated at buses two, six, and eight respectively and likewise three transformers and one shunt reactive power compensator are placed. The total active power demand as well as reactive power demand is $259 \mathrm{MW}$ and 73.5 MVar respectively.

Table 1: IEEE 14 Buses Model Load Parameters

\begin{tabular}{|c|c|c|}
\hline Load Bus & $\mathrm{P}(\mathrm{MW})$ & $\mathrm{Q}$ (Mvar) \\
\hline 2 & 21.7 & 12.7 \\
\hline 3 & 94.2 & 19.0 \\
\hline 4 & 47.8 & 3.9 \\
\hline 5 & 7.6 & 1.6 \\
\hline
\end{tabular}

\begin{tabular}{|c|c|c|}
\hline 6 & 11.2 & 7.5 \\
\hline 9 & 29.5 & 16.6 \\
\hline 10 & 9.0 & 5.8 \\
\hline 11 & 3.5 & 1.8 \\
\hline 12 & 6.1 & 1.6 \\
\hline 13 & 13.5 & 5.8 \\
\hline 14 & 14.9 & 5.0 \\
\hline
\end{tabular}

\subsection{Reactive Power Dispatch without New DG}

Many times, there would be no significant enhancement on the optimization results after process of iterations go on, the value of weight also will be dropped to 04 from 0.9 . The evry particle position is defined by a nine dimensional space which is represented by fig. 2. The individual population for the PSO algorithm is chosen as 50 . In general the population is chosen more than 4 times for the good optimization results in the literature. Initial inertia is chosen to be 0.9 and it is reduced to 0.4 for final iteration with step size decrement relative to number of iterations. Maximum number of iterations is taken as 200 as it is observed that our solution is not convergent for 150 iterations. Similarly initial acceleration constant is chosen as 2.0 and maximum velocity is chosen as 0.1 as the acceleration is 2.0 it should not change abruptly because we have to compute 200 iteration with 50 particles each.

\begin{tabular}{|l|l|l|l|l|l|l|l|l|}
\hline V1 & V2 & V3 & V6 & V8 & T1 & T2 & T3 & S9 \\
Fig. 2: Particle matches
\end{tabular}

In Fig. 2, V indicates the magnitude of voltage at the slack bus or $\mathrm{PV}$ bus, $\mathrm{T}$ indicates the transformer tap setting point, and S9 represents injection of reactive power at bus 9. Whenever optimization procedure takes place, every particle position will be continuously modified until reaching the stopping criteria.

Fig. 3 represents the without installing DG with optimization procedure of reactive power dispatch. Intial optimization process, the particles positions are selected randomly. At this time, the global active power loss is $13.5 \mathrm{MW}$. After updation of positions

of particles continually near the global finest result, real power loss becomes reducing. Once completion of hundred repetitions, no significant enhancement can be found and the real power loss converged to $12.36 \mathrm{MW}$ finally.

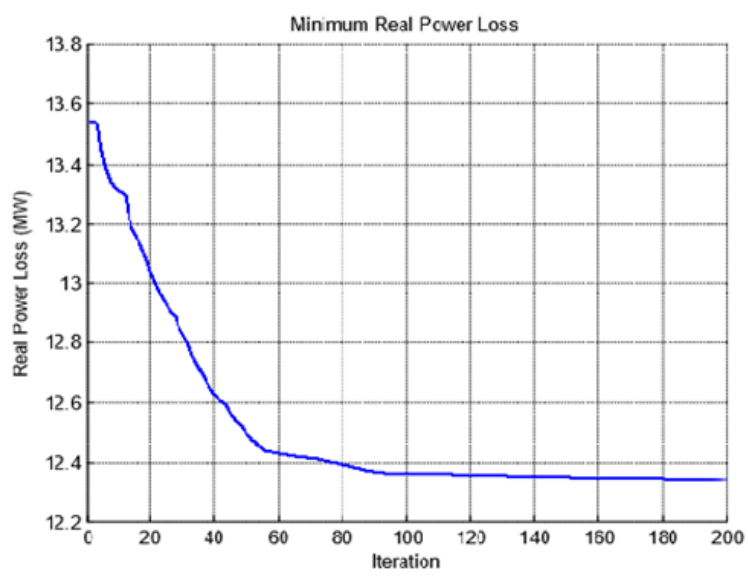

Fig. 3: Loss Decline Method

Table 2: Comparison of the Real Power Loss at Every Division

\begin{tabular}{|l|l|l|}
\hline $\begin{array}{l}\text { Branch } \\
\text { Number }\end{array}$ & $\begin{array}{l}\text { Before Optimization } \\
(\mathrm{MW})\end{array}$ & $\begin{array}{l}\text { After } \\
(\mathrm{MW})\end{array}$ \\
\hline $1-2$ & 4.298 & 3.907 \\
\hline $1-5$ & 2.763 & 2.552 \\
\hline $2-3$ & 2.323 & 2.147 \\
\hline $2-4$ & 1.677 & 1.546 \\
\hline $2-5$ & 0.904 & 0.828 \\
\hline $3-4$ & 0.373 & 0.347 \\
\hline
\end{tabular}




\begin{tabular}{|l|l|l|}
\hline $4-5$ & 0.514 & 0.462 \\
\hline $4-7$ & 0 & 0 \\
\hline $4-9$ & 0 & 0 \\
\hline $5-6$ & 0 & 0 \\
\hline $6-11$ & 0.055 & 0.055 \\
\hline $6-12$ & 0.072 & 0.073 \\
\hline $6-13$ & 0.212 & 0.213 \\
\hline $7-8$ & 0 & 0 \\
\hline $7-9$ & 0 & 0 \\
\hline $9-10$ & 0.013 & 0.013 \\
\hline $9-14$ & 0.116 & 0.120 \\
\hline $10-11$ & 0.013 & 0.013 \\
\hline $12-13$ & 0.006 & 0.006 \\
\hline $13-14$ & 0.054 & 0.053 \\
\hline
\end{tabular}

\subsection{Reactive Power Dispatch with New DG}

The alternative case study defines the introducing separate DG towards the IEEE 14 buses arrangement besides optimizing the reactive power of the arrangement via PSO. Gust drive, Solar Photovoltaic and Micro-turbine arrangements are preferred as substitute source to conventional DG unit. Proposed scheme implemented by installing Enercon E82 Gust drive instead of DG. Installed gust drive acts as direct-drive synchronous generator having the capacity of $200 \mathrm{KW}$. This arrangement operates at rated power in alternative case study.

If the gust drive installed at PQ bus, to modify the amount of reactive as well as real power to the innovative parameters, voltage level of the newly established DG bus ought to be preserved as newly regulating variable, which illustrates in fig. 4 .

\begin{tabular}{|l|l|l|l|l|l|l|l|l|l|}
\hline V1 & V2 & V3 & V6 & V8 & Vr & T1 & T2 & T3 & S9 \\
\hline
\end{tabular} Fig. 4: Coordinates of the Particles

Where $\mathrm{Vr}$ represents voltage magnitude of the new DG.

With addition of new DG, the load bus changes to a new generator bus. So system parameters bus data, generator data are changed. These changes are the combined with a 14 bus data case file and produce a new 14 bus data. This data is passed to PSO along with coordinates of the particle. PSO would then initialize for these 10 variables (fig.4) and send it to Newton Raphson (NR) technique using MATPOWER to find the load flow of system with losses. As the sum of the losses determine through NR method indicates fitness of particle, the best particle solution provides least losses with different voltages and tap setting at various points of IEEE 14 bus system providing optimal power flow with least real power losses.

Table 3: Real Power Loss Comparison

\begin{tabular}{|l|l|l|l|l|}
\hline Branch & $\begin{array}{l}\text { Before } \\
\text { optimization } \\
\text { (MW) }\end{array}$ & $\begin{array}{l}\text { Optimization } \\
\text { without } \\
\text { DG(MW) }\end{array}$ & $\begin{array}{l}\text { Optimization } \\
\text { with } \\
\text { DG(MW) }\end{array}$ & $\begin{array}{l}\text { Percentage } \\
\text { improvement } \\
(\%)\end{array}$ \\
\hline $1-2$ & 4.298 & 3.907 & 3.826 & 10.9819 \\
\hline $1-5$ & 2.763 & 2.552 & 2.517 & 8.9034 \\
\hline $2-3$ & 2.323 & 2.147 & 2.051 & 11.7090 \\
\hline $2-4$ & 1.677 & 1.546 & 1.531 & 8.7060 \\
\hline $2-5$ & 0.904 & 0.828 & 0.818 & 9.5133 \\
\hline $3-4$ & 0.373 & 0.347 & 0.309 & 17.1582 \\
\hline $4-5$ & 0.514 & 0.462 & 0.461 & 10.3113 \\
\hline $4-7$ & 0 & 0 & 0 & 0 \\
\hline $4-9$ & 0 & 0 & 0 & 0 \\
\hline $5-6$ & 0 & 0 & 0 & 0 \\
\hline $6-11$ & 0.055 & 0.055 & 0.038 & 30.9091 \\
\hline $6-12$ & 0.072 & 0.073 & 0.068 & 5.5556 \\
\hline $6-13$ & 0.212 & 0.213 & 0.194 & 8.4906 \\
\hline $7-8$ & 0 & 0 & 0 & 0 \\
\hline $7-9$ & 0 & 0 & 0 & 0 \\
\hline $9-10$ & 0.013 & 0.013 & 0.019 & -46.1538 \\
\hline $9-14$ & 0.116 & 0.120 & 0.135 & -16.3793 \\
\hline & & & & \\
\hline
\end{tabular}

\begin{tabular}{|l|l|l|l|l|}
\hline $10-11$ & 0.013 & 0.013 & 0.006 & 53.8462 \\
\hline $12-13$ & 0.006 & 0.006 & 0.005 & 16.6666 \\
\hline $13-14$ & 0.054 & 0.053 & 0.039 & 27.7777 \\
\hline
\end{tabular}

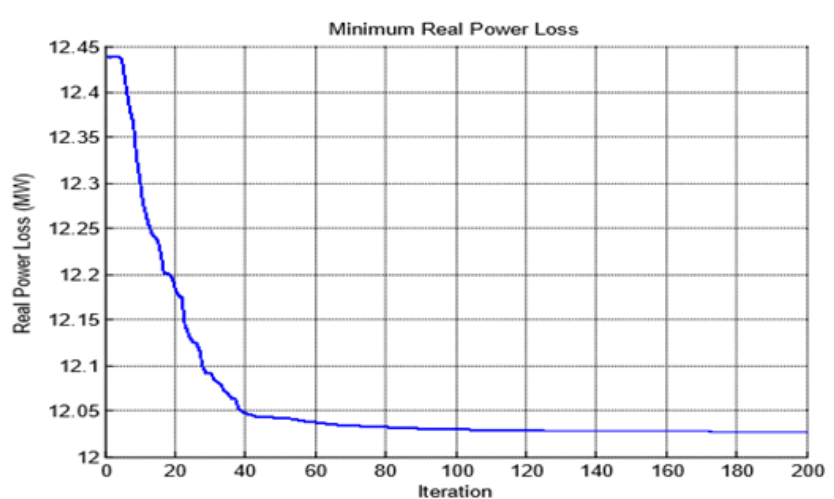

Fig. 5: Loss Reduction Process when the new DG is installed at Bus 3

Figure: 5 shows the optimal procedure of the suggested strategy, when newly gust drive is established at bus number 3 .

Therefore primary active power loss of the coordination is nearly 12.45 MW. The elements flinch to meet when conduction takes eighty iterations. Lastly entire power loss of the arrangement is 12.017 MW

\section{Conclusion:}

Reactive power dispatch is a nonlinear advancement issue, which comprises constant as well as distinct regulating factors. PSO is an empirical universal optimal type algorithm that retains high efficacy and sturdiness. PSO is fewer delicate to the complication of impartial tasks. Thus shows the substantial prospective for resolving reactive power dispatch harms. This article utilizes the IEEE 14 buses arrangement as the assessment scheme. PSO techniques as well as MATPOWER 5.1 instrument package are tested to diminish real power loss in power networks. Reactive power dispatch methodology can expressively diminish the power loss in power system network and this scheme is cost-effective, simply engaged in actual life. PSO process appearances the tremendous penetrating capability for determining nonlinear optimum complications. Apply PSO process to discourse the reactive power dispatch difficulties are technical viable and attain substantial profitable paybacks. Developed MATPOWER 5.1 is presented to compute power stream and accomplish the fairness restrictions in PSO based reactive power communication. Exactness of consequences as well sturdiness of the program also enriched.

\section{References}

[1] H. Dommel and W. Tinney. Optimal power flow solutions," IEEE Transactions on Power Apparatus and Systems. Vol. PAS-87, pp. 1866-1876, Oct 1968.

[2] H. Yoshida, K. Kawata, Y. Fukuyama, S. Takayama, and Y. Nakanishi. A particle swarm optimization for reactive power and voltage control considering voltage security assessment. IEEE Transactions on Power Systems, vol. 15, pp. 1232-1239, Nov 2000

[3] D. B. Das and C. Patvardhan. Reactive power dispatch with a hybrid stochastic search technique. International Journal of Electrical Power and Energy Systems, vol. 24, no. 9, pp. 731 - 736, 2002.

[4] M. Martinez-Rojas, A. Sumper, O. Gomis-Bellmunt, and A. SudriàAndreu. Reactive power dispatch in wind farms using particle swarm optimization technique and feasible solutions search. Applied Energy, vol. 88, no. 12, pp. 4678 - 4686, 2011.

[5] Y. Amrane, M. Boudour, A. A. Ladjici, and A. Elmaouhab Optimal \{VAR\} control for real power loss minimization using differential evolution algorithm. International Journal of Electrical Power and Energy Systems, vol. 66, pp. 262 - 271, 2015. 
[6] Y.-Y. Hong, F.-J. Lin, Y.-C. Lin, and F.-Y. Hsu. Chaotic pso-based var control considering renewables using fast probabilistic power flow. IEEE Transactions on Power Delivery, vol. 29, pp. 16661674, Aug 2014

[7] Aggelos S. Bouhouras, Kallisthenis I. Sgouras, Paschalis A Gkaidatzis and Dimitris P. Labridis. Optimal active and reactive nodal power requirements towards loss minimization under reverse power flow constraint defining DG type. International Journal of Electrical Power and Energy Systems, Vol. 78, pp. 445-454, June 2016.

[8] B. Kanna and S. N. Singh. Towards reactive power dispatch within a wind farm using hybrid pso. International Journal of Electrical Power and Energy Systems, vol. 69, pp. 232 - 240, 2015.

[9] N. Acharya, P. Mahat, and N. Mithulananthan. An analytical approach for DG allocation in primary distribution network. International Journal of Electrical Power and Energy Systems, vol. 28, no. 10, pp. 669-678, Dec.2006.

[10] B. Zhao, C. Guo, and Y. Cao. A multiagent-based particle swarm optimization approach for optimal reactive power dispatch. IEEE Transactions on Power Systems, vol. 20, pp. 1070-1078, May 2005.

[11] R. P. Singh, V. Mukherjee, and S. Ghoshal. Optimal reactive power dispatch by particle swarm optimization with an aging leader and challengers. Applied Soft Computing, vol. 29, pp. 298 - 309, 2015.

[12] Satish kansal, Vishal Kumar and BarjeevTyagi. Optimal placement of different type of DG sources in distribution networks. Electrical power and Energy Systems, vol. 53, pp.752-760, 2013.

[13] L. Srivastava and H. Singh. Hybrid multi-swarm particle swarm optimisation based multi-objective reactive power dispatch," IET Generation, Transmission Distribution, vol. 9, no. 8, pp. 727-739, 2015.

[14] Leeton U, Uthitsunthorn D, Kwannetr U, Sinsuphun N, Kulworawanichpong T. Power loss minimization using optimal power flow based on particle swarm optimization. Proceedings of IEEE Conference on Electrical Engineering/Electronics Computer Telecommunications and Information Technology (ECTI-CON), pp. 440-444, 2010.

[15] Z. L. Gaing. Particle swarm optimization to solving the economic dispatch considering the generator constraints. IEEE Transactions on Power Systems, vol. 18, no. 3, pp. 1187-1195, August 2003.

[16] J. Vlachogiannis and K. Lee. A comparative study on particle swarm optimization for optimal steady-state performance of power systems. IEEE Transactions on Power Systems, vol. 21, pp. 1718 1728, Nov 2006.

[17] J. Kennedy, R.C. Eberhart. Particle Swarm Optimization. Proceedings of IEEE Conference on Neural Networks, IV, Piscataway, NJ, pp. 1942-1948,1995. 